\title{
OPEN A search for modifying genetic factors in CHEK2:C.1100delC breast cancer patients
}

\author{
Camilla Wendt ${ }^{1 凶}$, Taru A. Muranen ${ }^{2}$, Lotta Mielikäinen ${ }^{2}$, Jessada Thutkawkorapin ${ }^{3}$, \\ Carl Blomqvist ${ }^{4}$, Xiang Jiao ${ }^{3}$, Hans Ehrencrona ${ }^{5}$, Emma Tham $^{3}$, Brita Arver 6 , Beatrice Melin ${ }^{7}$, \\ Ekaterina Kuchinskaya ${ }^{8}$, Marie Stenmark Askmalm ${ }^{8}$, Ylva Paulsson-Karlsson ${ }^{9}$, \\ Zakaria Einbeigi ${ }^{10}$, Anna von Wachenfeldt Väppling ${ }^{1}$, Eija Kalso ${ }^{11}$, Tiina Tasmuth ${ }^{11}$, \\ Anne Kallioniemi ${ }^{12}$, Kristiina Aittomäki ${ }^{13}$, Heli Nevanlinna ${ }^{2}$, Åke Borg ${ }^{14}$ \& Annika Lindblom ${ }^{3}$
}

The risk of breast cancer associated with CHEK2:c.1100delC is 2-threefold but higher in carriers with a family history of breast cancer than without, suggesting that other genetic loci in combination with CHEK2:c.1100delC confer an increased risk in a polygenic model. Part of the excess familial risk has been associated with common low-penetrance variants. This study aimed to identify genetic loci that modify CHEK2:c.1100delC-associated breast cancer risk by searching for candidate risk alleles that are overrepresented in CHEK2:c.1100delC carriers with breast cancer compared with controls. We performed whole-exome sequencing in 28 breast cancer cases with germline CHEK2:c.1100delC, 28 familial breast cancer cases and 70 controls. Candidate alleles were selected for validation in larger cohorts. One recessive synonymous variant, rs 16897117 , was suggested, but no overrepresentation of homozygous CHEK2:c.1100delC carriers was found in the following validation. Furthermore, 11 non-synonymous candidate alleles were suggested for further testing, but no significant difference in allele frequency could be detected in the validation in CHEK2:c.1100delC cases compared with familial breast cancer, sporadic breast cancer and controls. With this method, we found no support for a CHEK2:c.1100delC-specific genetic modifier. Further studies of CHEK2:c.1100delC genetic modifiers are warranted to improve risk assessment in clinical practice.

Breast cancer aggregates in families and has a considerable inherited component. Approximately $20 \%$ of the genetic risk for breast cancer is explained by pathogenic mutations in the high-penetrance genes $B R C A 1, B R C A 2$, TP53, STK11 and PTEN ${ }^{1}$. Other rare, intermediate-risk variants, such as PALB2, CHEK2 and ATM account for about $5 \%$ of the inherited risk $^{2,3}$ and common low-risk variants for another $18-19 \%{ }^{4-6}$.

Checkpoint kinase 2 is a protein product of the CHEK2 gene that localizes to chromosome 22q12.1. It is part of the network that responds to DNA damage in order to maintain genomic integrity ${ }^{7}$. The protein-truncating variant CHEK2:c.1100delC is associated with a two-threefold risk of breast cancer ${ }^{8,9}$. In women with familial aggregation of breast cancer, the risk is even higher. An odds ratio of up to 4.8 has been seen in women with a family history of breast cancer, which is equivalent to a $37 \%$ cumulative risk of breast cancer by the age of

\footnotetext{
${ }^{1}$ Department of Clinical Science and Education, Karolinska Institutet, Södersjukhuset, Stockholm, Sweden. ${ }^{2}$ Department of Obstetrics and Gynecology, Helsinki University Hospital, University of Helsinki, Helsinki, Finland. ${ }^{3}$ Department of Molecular Medicine and Surgery, Karolinska Institutet, Solna, Stockholm, Sweden. ${ }^{4}$ Department of Oncology, Helsinki University Hospital, University of Helsinki, Helsinki, Finland. ${ }^{5}$ Department of Clinical Genetics and Pathology, Office for Medical Services, Region Skåne, Lund, Sweden. ${ }^{6}$ Department of Oncology-Pathology, Karolinska Institutet, Solna, Stockholm, Sweden. ${ }^{7}$ Department of Radiation Sciences, Oncology, Umeå University, Umeå, Sweden. ${ }^{8}$ Department of Clinical Genetics, Department of Clinical Experimental Medicine, Linköping University, Linköping, Sweden. ${ }^{9}$ Department of Immunology, Genetics and Pathology, Uppsala University, Uppsala, Sweden. ${ }^{10}$ Department of Oncology, Sahlgrenska University Hospital, 41345 Göteborg, Sweden. ${ }^{11}$ Department of Anaesthesiology, Intensive Care, and Pain Medicine, Helsinki University Hospital and University of Helsinki, Helsinki, Finland. ${ }^{12}$ TAYS Cancer Centre and Faculty of Medicine and Health Technology, Tampere University; Fimlab Laboratories, Tampere University Hospital, Tampere, Finland. ${ }^{13}$ Department of Medical and Clinical Genetics, University of Helsinki, Helsinki, Finland. ${ }^{14}$ Department of Oncology and Pathology, Department of Clinical Sciences Lund, Lund University, Medicon Village, Lund, Sweden. ${ }^{\square}$ email: camilla.wendt@sll.se
} 
70 years $^{8-10}$. In addition, the c.1100delC allele has been associated with younger age at onset, a threefold increased risk of a second breast cancer, as well as a worse prognosis among women with oestrogen receptor-positive cancer $^{9,11,12}$.

The considerably higher risk in women with a family history of breast cancer is in accordance with the suggested polygenic model where several susceptibility loci together confer a multiplicative effect on breast cancer risk $^{13,14}$. The fact that the model also can be applied to CHEK2:c.1100delC carriers is supported by a study of low-risk breast cancer variants in 34000 women with and without a family history of breast cancer. A polygenic risk score (PRS) that was based on the combined risk of 74 low risk variants was calculated. The result suggested that the polygenic risk score could be used to stratify risk in c.1100delC carriers and that the low-risk variants explained a part of the familial risk. The authors estimated that 20\% of CHEK2:c.1100delC carriers with the highest PRS had an estimated lifetime breast cancer risk of $>30 \%$. Correspondingly, $20 \%$ of carriers with the lowest PRS had an estimated lifetime risk of $14 \%$ which is close to the average population risk ${ }^{15}$. A synergistic effect between low-risk variants and $B R C A 1$ and $B R C A 2$ mutations has also been shown ${ }^{16}$. The risk for mutation carriers being affected is thus modified by other genetic variants and family history in addition to lifestyle factors. A risk prediction model, the Breast and Ovarian Analysis of Disease Incidence and Carrier Estimation Algorithm (BOADICEA) has been developed to calculate the lifetime risk of breast cancer, including carriers of a moderate-penetrance allele such as CHEK2:c.1100delC. The BOADICEA model allows risk stratification for established genetic and non-genetic risk factors ${ }^{17}$. Still, other causative gene variants possibly remain to be identified, since the previously identified low-, intermediate-, and high-risk genes cover less than half of the estimated heritable component. Characterising factors that increase the risk in carriers of moderate-risk alleles is important, in order to identify the high-risk group that benefits most from preventive interventions. In this study, we used whole-exome sequencing of a CHEK2:c.1100delC positive cohort with familial breast cancer, to identify putative risk modifying alleles. In the first phase we aimed to find candidate risk alleles for further validation in the second phase with larger cohorts of CHEK2:c.1100delC positive cases and controls.

\section{Results}

We performed whole-exome sequencing in 28 breast cancer cases with germline CHEK2:c.1100delC, 28 familial breast cancer cases and 70 controls. Candidate alleles were selected for validation in larger cohorts (Fig. 1).

Recessive variants. We analysed the exome sequencing data for a discovery of rare homozygous variants in CHEK2:c.1100C carriers, to identify risk alleles with recessive inheritance pattern. Only one variant was suggested, rs16897117. Among the 28 CHEK2 carriers, there were 3 patients homozygous for rs 16897117 , whereas among the non-carrier breast cancer cases or healthy controls, there were no rs 16897117 homozygotes. We set up to test the hypothesis of rs1689711 being a CHEK2:c.1100C risk modifier in larger sample collections, starting with 67 CHEK2 patients, as well as 688 non-carrier breast cancer cases and 246 healthy controls. This study confirmed the skewed allele distribution, with fewer individuals heterozygous for rs16897117 among the CHEK2 patients than among non-carrier patients or healthy controls. In a case-only analysis, the odds ratio between rs16897117 rare allele (A) and CHEK2:c.1100delC was 0.46 (95\% confidence interval CI 0.17-1.04, $P$ 0.053 (Table 1: SWEA1).

Next, we did another follow-up using 45 CHEK2 carriers plus 87 familial breast cancer patients and 47 controls from the Swedish cohorts. None of the CHEK2 carriers or the familial breast cancer patients were found to be homozygous for the rs16897117 variant. The only two homozygous individuals of this follow-up were identified in the control group. No skewness in allele distribution was observed in any of these groups (Table 1: SWEA2). The results seemed less clear, but to resolve this, we tested the association between rs16897117 and c.1100delC in a Finnish population, where the c.1100delC allele has a relatively high, $1.2 \%$, frequency ${ }^{18}$. Genotyping of three independent patient series identified a single c.1100delC carrier patient, who was homozygous for rs16897117. The skewed allele distribution for rs16897117 was observed in the Helsinki cohorts, but not in the Tampere cohort. A study-stratified OR for association between rs16897117 and c.1100delC, combining all cohorts from Sweden and Finland, was 0.69 (95\% CI 0.46-1.03, P 0.073), encouraging further analysis.

Finally, the genotype data for rs 16897117 and c.1100delC were obtained from the OncoArray project of the Breast Cancer Association Consortium ${ }^{5}$. The availability of a good number of healthy c.1100delC carriers in the consortium data enabled a proper interaction analysis for c.1100delC, rs16897117, and breast cancer risk. In the BCAC data, there was no allelic imbalance between rs16897117 and c.1100delC (Table 2). A likelihood-ratio test comparing a breast cancer risk model with c.1100delC-rs16897117 interaction term with a plain model with c.1100delC and rs16897117 as independent risk factors did not support rs16897117 as a dosage-dependent risk modifier for c.1100delC carriers (Table 3). The BCAC data included four c.1100delC carriers, who were homozygous for rs 16897117 . These were all breast cancer cases, but the sample counts were too low for a reliable analysis.

Coding non-synonymous candidate variants. In the discovery phase, exome sequencing data were analysed with a set of criteria in search of CHEK2:c.1100delC candidate variants. Fourteen non-synonymous variants were subject for testing, but only 11 were analysed due to technical issues with TaqMan probes (Table 4). The 11 missense variants detected in the CHEK2:c.1100delC carriers were evaluated in the validation phase. None of the variants could be replicated with similar patterns as in the discovery phase (Table 5). Thus, none was suggested to be a modifier of breast cancer risk in CHEK2:c.1100delC carriers. 


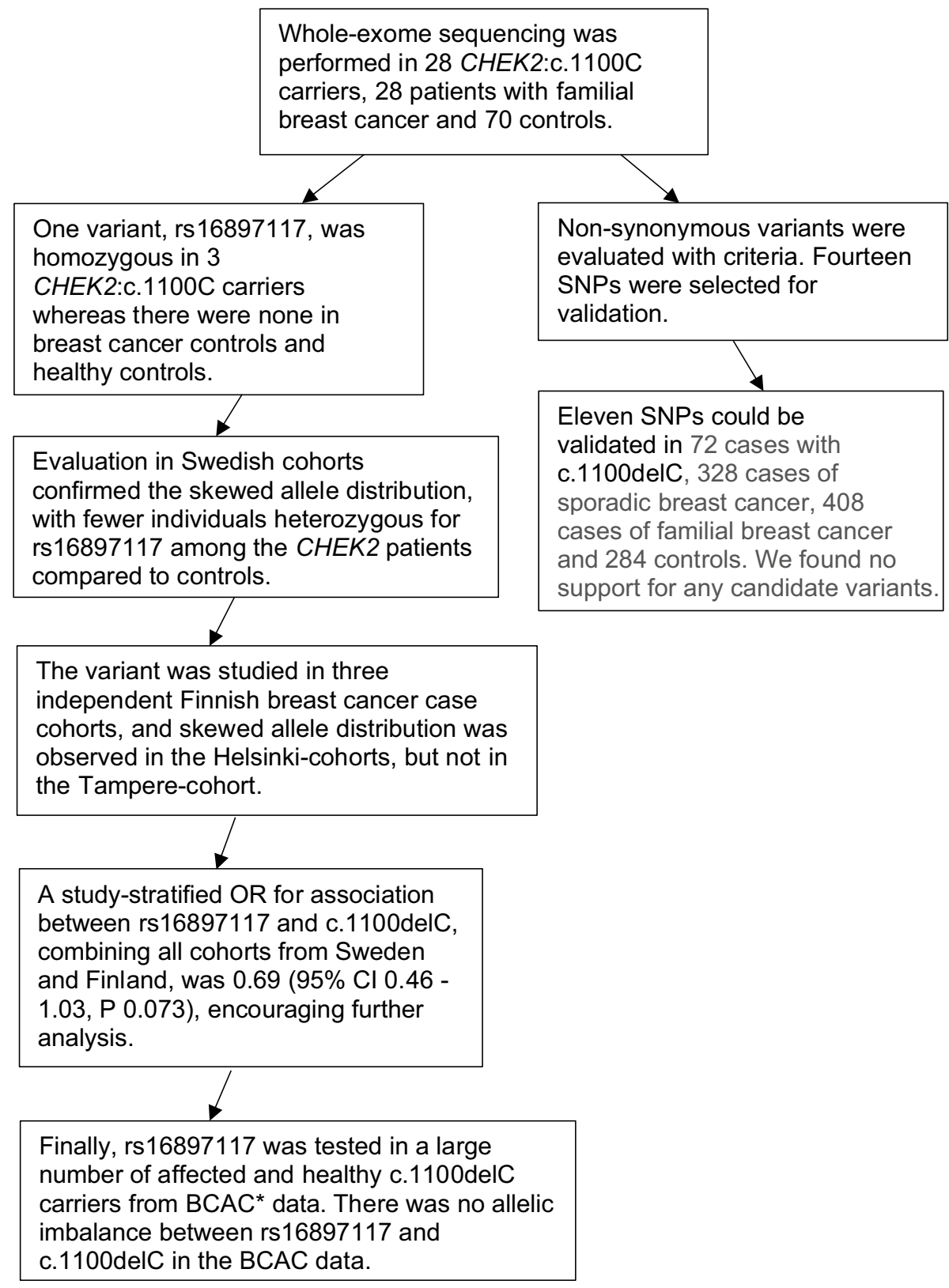

Figure 1. Flowchart describing the working process of evaluating genotype data in search of variants that specifically modify breast cancer risk in CHEK2:c.1100del carriers. ${ }^{\star}$ Breast Cancer Association Consortium.

\section{Discussion}

We aimed to identify candidate risk variants that specifically modify risk in CHEK2:c.1100delC carriers through whole-exome sequencing of a small number of samples followed by validation in a case-control association study. No CHEK2:c.del1100C-specific candidate variants could be identified. Previously identified variants that modify breast cancer risk in CHEK2:c.1100delC carriers are also risk variants in the general breast cancer population. The common low-risk variants that predispose to breast cancer have also shown synergistic effects with CHEK2 ${ }^{14}$. To our knowledge, no other genetic modifiers of CHEK2:c.1100delC have been suggested. Previously identified common alleles, associated with breast cancer in the general population have also been shown to modify risk in $B R C A 1$ and $B R C A 2$ mutation carriers, in a subtype specific manner ${ }^{16}$. A recent GWAS identified several novel loci that were associated with at least one tumour feature (ER-status, progesterone receptor status, tumour grade, human epidermal growth factor 2 receptor) and also loci that differed by the molecular subtype, luminal or non-luminal, of breast cancer ${ }^{19}$. The observations imply that tumour features should be taken into account when searching for candidate variants in CHEK2:c.del1100C carriers. Several loci that specifically modify risk in $B R C A 1$ and $B R C A 2$ carriers have also been found ${ }^{16,20-29}$. These are all low-risk susceptibility alleles identified through testing of candidates from breast cancer genome-wide association studies in $B R C A 1 / 2$ mutation carriers and through fine-mapping of candidate regions. 


\begin{tabular}{|c|c|c|c|c|}
\hline Cohort & $\begin{array}{l}\text { rs16897117 in } \\
\text { non-carriers } \\
\text { GG-GA-AA }\end{array}$ & $\begin{array}{l}\text { rs } 16897117 \text { in c.1100delC carriers } \\
\text { GG-GA-AA }\end{array}$ & OR $[95 \% \mathrm{CI}]$ & $P$ \\
\hline \multirow{2}{*}{ SWEA1 } & $549-138-1$ & $60-5-2$ & $0.46[0.17-1.04]$ & 0.053 \\
\hline & $(80 \%-20 \%-0.1 \%)$ & $(90 \%-7 \%-3 \%)$ & & \\
\hline \multirow{2}{*}{ SWEA2 } & $73-14-0$ & $34-11-0$ & $1.68[0.62-4.47]$ & 0.25 \\
\hline & $(84 \%-16 \%-0 \%)$ & $(76 \%-24 \%-0 \%)$ & & \\
\hline \multirow{2}{*}{ Helsinki1-unselected } & $1432-232-9$ & $44-4-0$ & $0.54[0.14-1.50]$ & 0.30 \\
\hline & $(86 \%-14 \%-0.5 \%)$ & $(92 \%-8 \%-0 \%)$ & & \\
\hline \multirow{2}{*}{ Helsinki1—additional familial } & $603-99-3$ & $45-4-1$ & $0.66[0.20-1.71]$ & 0.53 \\
\hline & $(86 \%-14 \%-0.4 \%)$ & $(90 \%-8 \%-2 \%)$ & & \\
\hline \multirow{2}{*}{ Helsinki2 } & $841-119-5$ & $26-2-0$ & $0.52[0.06-2.13]$ & 0.57 \\
\hline & $(87 \%-12 \%-0.5 \%)$ & $(93 \%-7 \%-0 \%)$ & & \\
\hline \multirow{2}{*}{ Tampere } & $564-87-1$ & $12-2-0$ & $1.10[0.11-4.92]$ & 1.00 \\
\hline & $(87 \%-13 \%-0.2 \%)$ & $(86 \%-14 \%-0 \%)$ & & \\
\hline Combined & & & $0.69[0.46-1.03]$ & 0.073 \\
\hline
\end{tabular}

Table 1. Rs16897117 association with CHEK2:c.1100delC in a case-only analysis.

\begin{tabular}{|l|l|l|}
\hline & $\begin{array}{l}\text { rs16897117 in } \\
\text { non-carriers } \\
\text { GG-GA-AA }\end{array}$ & $\begin{array}{l}\text { rs16897117 in c.1100delC carriers } \\
\text { GG-GA-AA }\end{array}$ \\
\hline \multirow{2}{*}{ Breast cancer cases } & $11,220-2247-111$ & $147-38-4$ \\
\cline { 2 - 3 } & $(83 \%-17 \%-1 \%)$ & $(78 \%-20 \%-2 \%)$ \\
\hline \multirow{2}{*}{ Healthy controls } & $17,561-3564-180$ & $124-27-0$ \\
\cline { 2 - 3 } & $(82 \%-17 \%-1 \%)$ & $(82 \%-18 \%-0 \%)$ \\
\hline
\end{tabular}

Table 2. BCAC breast cancer cases and healthy controls with available data on CHEK2:c.1100delC and rs16897117 from the OncoArray project.

\begin{tabular}{|l|l|l|l|}
\hline & Plain model (OR) & Interaction model (OR) & P.LR \\
\hline rs16897117 & $1.00[0.95-1.06]$ & $1.00[0.94-1.05]$ & 0.26 \\
\hline c.1100delC & $2.03[1.63-2.54]$ & $1.92[1.50-2.45]$ & \\
\hline Interaction term & & $1.34[0.80-2.28]$ & \\
\hline
\end{tabular}

Table 3. The breast cancer risk associated with CHEK2:c.1100delC and rs16897117 in the BCAC data. The models were adjusted for BCAC study and 10 principal components.

Future studies of CHEK2:c.1100delC modifying candidates could be done with more loose criteria in the discovery phase to increase the probability of finding good candidates for further testing. In accordance with previous findings, gene-specific modifiers are likely to be common low-risk variants. CHEK2:c.1100delC-specific modifiers may then rather be identified through large-scale genome-wide association studies. With this method, we found no support for a CHEK2:c.1100delC-specific genetic modifier. More studies of CHEK2:c.1100delC genetic modifiers are therefore warranted to improve risk assessment in clinical practice.

\section{Methods}

In order to identify candidate variants, we conducted a discovery phase, where whole-exome sequencing was performed in 28 CHEK2:c. $1100 \mathrm{delC}$ carriers with familial breast cancer, another 28 familial breast cancer patients and 70 healthy controls (spouses of colorectal cancer patients) from the Swedish cohorts. Candidate variants were validated in larger cohorts (Fig. 1).

Sample preparation, discovery phase. Genomic DNA was subjected to whole-exome sequencing at the National Genomics Infrastructure in Uppsala, Sweden. Exome-enriched sequencing libraries were prepared using the Agilent SureSelectXT Human All Exon V5 XT2 + UTR kit (Agilent, Santa Clara, California, US). Cluster generation and 125 cycle paired-end sequencing was performed using the Illumina HiSeq 2500 system and v4 sequencing chemistry (Illumina, San Diego, California, US). Next-generation sequencing was performed at SciFiLab, University of Uppsala. 


\begin{tabular}{|c|c|c|c|c|c|c|c|c|c|c|c|c|}
\hline Chr & Variant type & SNP & Gene & $\begin{array}{l}\text { 1000g2014 } \\
\text { oct eur }\end{array}$ & 249 Swedes & 200 Danes & ExAC NFE & $\begin{array}{l}\text { CHEK2/ } \\
\text { CRC }\end{array}$ & $\begin{array}{l}\text { CHEK2/ } \\
\text { FBC }\end{array}$ & CRC MAF & FBC MAF & $\begin{array}{l}\text { CHEK2 } \\
\text { MAF }\end{array}$ \\
\hline 1 & SNV & rs2297809 & CYP4B1 & 0.1302 & 0.1456 & 0.12 & 0.1457 & 2.07 & 1.65 & 0.1207 & 0.1518 & 0.25 \\
\hline 1 & SNV & rs4926600 & CYP4A22 & 0.0805 & 0.0984 & - & 0.0868 & 3.06 & 1.29 & 0.0641 & 0.1518 & 0.1964 \\
\hline 2 & SNV & rs17860405 & CASP10 & 0.0417 & 0.0382 & 0.0225 & 0.0409 & 7.51 & 1.80 & 0.0214 & 0.0893 & 0.1607 \\
\hline 3 & SNV & rs34492126 & DLG1 & 0.0577 & 0.0542 & 0.0575 & 0.0535 & 2.35 & 1.64 & 0.0684 & 0.0982 & 0.1607 \\
\hline 5 & SNV & rs2287749 & ADAM19 & 0.1163 & 0.1426 & 0.14 & 0.1350 & 1.39 & 1.53 & 0.1667 & 0.1518 & 0.2321 \\
\hline 6 & SNV & rs811925 & PRDM1 & 0.2048 & 0.1767 & 0.1475 & 0.1758 & 2.04 & 1.17 & 0.1838 & 0.3214 & 0.375 \\
\hline 9 & SNV & rs34523498 & CDK5RAP2 & 0.0328 & 0.0361 & 0.02 & 0.0295 & 4.18 & 1.33 & 0.0256 & 0.0804 & 0.1071 \\
\hline 9 & SNV & rs41305617 & NOL8 & 0.0338 & 0.0221 & - & 0.0302 & 4.89 & 1.71 & 0.0219 & 0.0625 & 0.1071 \\
\hline 11 & SNV & rs8176786 & NELL1 & 0.0547 & 0.0582 & 0.06 & 0.0529 & 2.44 & 1.55 & 0.0513 & 0.0804 & 0.125 \\
\hline 11 & SNV & rs117739035 & SIGIRR & 0.0358 & 0.0482 & 0.045 & 0.0347 & 4.18 & 1.55 & 0.0299 & 0.0804 & 0.125 \\
\hline 12 & SNV & rs7962217 & VWF & 0.0507 & 0.0542 & 0.0625 & 0.0545 & 2.09 & 1.71 & 0.513 & 0.0625 & 0.1071 \\
\hline 15 & SNV & rs35932273 & LTK & 0.0268 & 0.0321 & 0.01 & 0.0285 & 4.18 & 1.50 & 0.0256 & 0.0714 & 0.1071 \\
\hline 16 & SNV & rs152451 & PALB2 & 0.0934 & 0.0683 & 0.0575 & 0.0955 & 5.53 & 0.93 & 0.0226 & 0.1339 & 0.125 \\
\hline 20 & SNV & rs34983477 & TP53RK & 0.0398 & 0.0502 & 0.0275 & 0.0473 & 2.09 & 1.20 & 0.0513 & 0.0893 & 0.1071 \\
\hline
\end{tabular}

Table 4. Variants selected in the discovery phase for further validation. SNV, single nucleotide variant; SNP, single nucleotide variant; MAF, minor allele frequency; FBC, familial breast cancer cohort; CRC, cohort of healthy spouses in colorectal cancer families. Column 5-8 display MAF for the reference databases described in methods. CHEK2 cohort of CHEK2:c.1100delC carriers.

Selection of non-synonymous candidate variants. After exome sequencing, all detected coding nonsynonymous variants in the CHEK2:c.1100delC carriers were evaluated. The cases of hereditary breast cancer and the healthy controls (spouses of cases with hereditary colon cancer) served as genotyping controls in the work of identifying candidate alleles. Only variants passing a set of criteria, described below, were selected for further evaluation. The criteria were as follows:

Allele frequency. Ratios of the allele frequencies of the variants were calculated. A ratio of 2.0 or more between CHEK2:c.1100delC cases and healthy controls and/or a ratio of 1.5 or more between CHEK2:c.1100delC cases and familial breast cancer cases was required.

Gene function. Genes/variants that were selected should display a function of a putative cancer driver gene when evaluated by online genome browser databases (OMIM, GeneCards) and scientific publications available on PubMed.

Reference databases. A more than $30 \%$ higher allele frequency in CHEK2:c.1100delC carriers compared with regional reference databases was required (ExAC non-Finnish population, 1000genome2014oct European, SweGen Variant Frequency Browser, exome sequencing data from 200 Danes $^{30}$ and anonymous exome data from a cohort of 249 controls from the Department of Clinical Genetics, Karolinska University Hospital).

Sequencing accuracy. Only variants with a sequencing accuracy of $65 \%$, or more, in all study groups were included. The variants passing the selection criteria were functionally annotated using the in silico tools SIFT, Polyphen2 HDIV/HVAR, LRT, MutationTaster, FATHMM, RadialSVM, LR, and MutationAssessor.

Validation of non-synonymous candidate variants. Eleven SNPs (rs2297809, rs17860405, rs8176786, rs34523498, rs117739035, rs34983477, rs152451, rs811925, rs7962217, rs34492126 and rs2287749) were genotyped using TaqMan SNP genotyping assay (Thermo Fisher Scientific, Waltham, Massachusetts, USA). rs35932273 was genotyped by Sanger sequencing following PCR. The candidates were validated in 72 cases with CHEK2:c. $1100 \mathrm{delC}, 328$ cases of sporadic breast cancer, 408 cases of familial breast cancer and 284 controls from the Swedish cohorts.

Genotyping of a recessive candidate allele. Exome sequencing data were analysed in search of recessive candidate variants in CHEK2:c.1100delC carriers. One recessive variant, rs16897117, was suggested, as among the 28 CHEK 2 carriers, there were 3 patients homozygous for rs16897117, whereas among the non-carrier breast cancer cases or healthy controls, there were no rs 16897117 homozygotes. The rs 16897117 was further evaluated in Swedish and Finnish cohorts and in data from the Breast Cancer Association Consortium, BCAC.

Swedish cohorts. The 28 samples from CHEK2:c.1100delC carriers analysed in the discovery phase were collected from the Department of Clinical Genetics, Karolinska University Hospital. A total of 112 samples from CHEK2:c.1100delC carriers were collected from the SWEA-study, a national Swedish collaboration aiming to study the prevalence of established breast cancer genes as well as to validate candidate genes and single nucleotide polymorphisms (SNPs) in Swedish women with familial breast- and ovarian cancer (72 and 112 samples for 


\begin{tabular}{|c|c|c|c|c|c|c|c|c|}
\hline Gene/rs number & Cohort & Heterozygous & Homozygous & Wild type & Samples & Allele frequency & \begin{tabular}{|l|} 
Odds ratio CI \\
$95 \%$
\end{tabular} & $P$ value \\
\hline \multirow{4}{*}{ PALB2 rs152451 } & CHEK2 & 6 & 0 & 64 & 70 & 0.043 & $\begin{array}{l}0.623[0.256- \\
1.518]\end{array}$ & 0.293 \\
\hline & Familial & 48 & 7 & 327 & 383 & 0.081 & $\begin{array}{l}1.228[0.792- \\
1.904]\end{array}$ & 0.357 \\
\hline & Sporadic & 31 & 4 & 262 & 297 & 0.066 & $\begin{array}{l}0.977[0.605- \\
1.579]\end{array}$ & 0.925 \\
\hline & \begin{tabular}{|l|} 
Controls \\
\end{tabular} & 27 & 3 & 216 & 246 & 0.067 & 1 & \\
\hline \multirow{4}{*}{ PRDM1 rs811925 } & CHEK2 & 26 & 1 & 44 & 71 & 0.197 & $\begin{array}{l}1.087[0.679- \\
1.739]\end{array}$ & 0.728 \\
\hline & Familial & 129 & 18 & 243 & 390 & 0.211 & $\begin{array}{l}1.187[0.895- \\
1.574]\end{array}$ & 0.232 \\
\hline & Sporadic & 103 & 12 & 200 & 315 & 0.201 & $\begin{array}{l}1.117[0.831- \\
1.503]\end{array}$ & 0.463 \\
\hline & Controls & 76 & 9 & 170 & 255 & 0.184 & 1 & \\
\hline \multirow{4}{*}{$\begin{array}{l}\text { ADAM19 } \\
\text { rs2287749 }\end{array}$} & CHEK2 & 18 & 2 & 50 & 70 & 0.157 & $\begin{array}{l}1.126[0.671- \\
1.891]\end{array}$ & 0.653 \\
\hline & Familial & 103 & 7 & 256 & 366 & 0.160 & $\begin{array}{l}1.149[0.837- \\
1.578]\end{array}$ & 1.149 \\
\hline & Sporadic & 71 & 3 & 230 & 304 & 0.127 & $\begin{array}{l}0.876[0.621- \\
1.236]\end{array}$ & 0.451 \\
\hline & Controls & 59 & 7 & 191 & 257 & 0.142 & 1 & \\
\hline \multirow{4}{*}{$\begin{array}{l}\text { CYP4B1 } \\
\text { rs2297809 }\end{array}$} & CHEK2 & 16 & 3 & 47 & 65 & 0.167 & $\begin{array}{l}1.005[0.600- \\
1.681]\end{array}$ & 0.985 \\
\hline & Familial & 81 & 14 & 255 & 350 & 0.156 & $\begin{array}{l}0.927[0.678- \\
1.266]\end{array}$ & 0.632 \\
\hline & Sporadic & 45 & 17 & 227 & 289 & 0.137 & $\begin{array}{l}0.795[0.569- \\
1.111]\end{array}$ & 0.179 \\
\hline & Controls & 63 & 10 & 177 & 250 & 0.166 & 1 & \\
\hline \multirow{4}{*}{ VWF rs7962217 } & CHEK2 & 6 & 0 & 64 & 70 & 0.043 & $\begin{array}{l}0.640[0.261- \\
1.566]\end{array}$ & 0.325 \\
\hline & Familial & 38 & 3 & 340 & 381 & 0.058 & $\begin{array}{l}0.876[0.545- \\
1.408]\end{array}$ & 0.583 \\
\hline & Sporadic & 21 & 3 & 279 & 303 & 0.044 & $\begin{array}{l}0.666[0.392- \\
1.133]\end{array}$ & 0.131 \\
\hline & Controls & 25 & 3 & 209 & 237 & 0.065 & 1 & \\
\hline \multirow{4}{*}{$\begin{array}{l}\text { CASP10 } \\
\text { rs17860405 }\end{array}$} & CHEK2 & 8 & 1 & 60 & 69 & 0.072 & $\begin{array}{l}1.078[0.520- \\
2.236]\end{array}$ & 0.840 \\
\hline & Familial & 25 & 0 & 342 & 367 & 0.034 & $\begin{array}{l}0.487[0.288- \\
0.823]\end{array}$ & 0.006 \\
\hline & Sporadic & 19 & 0 & 270 & 289 & 0.033 & $\begin{array}{l}0.469[0.265- \\
0.831]\end{array}$ & 0.008 \\
\hline & Controls & 27 & 4 & 228 & 259 & 0.067 & 1 & \\
\hline \multirow{4}{*}{$\begin{array}{l}\text { DLG1 } \\
\text { rs34492126 }\end{array}$} & CHEK2 & 8 & 0 & 63 & 71 & 0.056 & $\begin{array}{l}1.155[0.505- \\
2.642]\end{array}$ & 0.732 \\
\hline & Familial & 41 & 2 & 344 & 387 & 0.058 & $\begin{array}{l}1.194[0.713- \\
2.001]\end{array}$ & 0.499 \\
\hline & Sporadic & 34 & 0 & 266 & 300 & 0.057 & $\begin{array}{l}1.162[0.675- \\
2.001]\end{array}$ & 0.587 \\
\hline & Controls & 19 & 2 & 213 & 234 & 0.049 & 1 & \\
\hline \multirow{4}{*}{$\begin{array}{l}\text { CDK5RAP2 } \\
\text { rs34523498 }\end{array}$} & CHEK2 & 5 & 0 & 65 & 71 & 0.036 & $\begin{array}{l}1.125[0.405- \\
3.126]\end{array}$ & 0.791 \\
\hline & Familial & 24 & 0 & 358 & 382 & 0.031 & $\begin{array}{l}0.985[0.518- \\
1.874]\end{array}$ & 0.963 \\
\hline & Sporadic & 22 & 1 & 285 & 309 & 0.039 & $\begin{array}{l}1.231[0.647- \\
2.344]\end{array}$ & 0.526 \\
\hline & Controls & 14 & 1 & 236 & 251 & 0.032 & 1 & \\
\hline \multirow{4}{*}{$\begin{array}{l}\text { TP53RK } \\
\text { rs34983477 }\end{array}$} & CHEK2 & 10 & 0 & 60 & 70 & 0.071 & $\begin{array}{l}1.1330 .538- \\
2.386]\end{array}$ & 0.743 \\
\hline & Familial & 33 & 2 & 327 & 362 & 0.051 & $\begin{array}{l}0.793[0.481- \\
1.309]\end{array}$ & 0.363 \\
\hline & Sporadic & 30 & 2 & 255 & 287 & 0.059 & $\begin{array}{l}0.927[0.556- \\
1.546]\end{array}$ & 0.927 \\
\hline & Controls & 19 & 5 & 204 & 228 & 0.063 & 1 & \\
\hline
\end{tabular}




\begin{tabular}{|c|c|c|c|c|c|c|c|c|}
\hline Gene/rs number & Cohort & Heterozygous & Homozygous & Wild type & Samples & Allele frequency & $\begin{array}{l}\text { Odds ratio CI } \\
95 \%\end{array}$ & $P$ value \\
\hline \multirow{4}{*}{$\begin{array}{l}\text { SIGIRR } \\
\text { rs117739035 }\end{array}$} & CHEK2 & 5 & 0 & 65 & 70 & 0.0357 & $\begin{array}{l}0.784[0.293- \\
2.102]\end{array}$ & 0.628 \\
\hline & Familial & 45 & 0 & 349 & 394 & 0.057 & $\begin{array}{l}1.282[0.766- \\
2.147]\end{array}$ & 0.342 \\
\hline & Sporadic & 28 & 0 & 288 & 316 & 0.044 & $\begin{array}{l}0.982[0.558- \\
1.726]\end{array}$ & 0.948 \\
\hline & Controls & 23 & 0 & 232 & 255 & 0.045 & 1 & \\
\hline \multirow{4}{*}{ NELL1 rs8176786 } & CHEK2 & 3 & 1 & 46 & 53 & 0.031 & $\begin{array}{l}0.392[0.136- \\
1.131]\end{array}$ & 0.073 \\
\hline & Familial & 36 & 2 & 348 & 386 & 0.052 & $\begin{array}{l}0.546[0.340- \\
0.879]\end{array}$ & 0.011 \\
\hline & Sporadic & 33 & 2 & 264 & 297 & 0.055 & $\begin{array}{l}0.588[0.358- \\
0.967]\end{array}$ & 0.035 \\
\hline & Controls & 24 & 5 & 158 & 187 & \begin{tabular}{|l|}
0.091 \\
\end{tabular} & 1 & \\
\hline
\end{tabular}

Table 5. Odds ratios for the 11 validated candidate alleles in CHEK2:c.1100delC familial breast cancer and sporadic breast cancer. CI, confidence interval.

validation of non-synonymous variants and the recessive variant respectively). All CHEK2:c.1100delC carriers were previously affected by breast cancer except for two carriers who had been diagnosed with ovarian cancer. All cases of hereditary breast cancer were collected from the Department of Clinical Genetics, Karolinska University Hospital and had previously received counselling and screened negative for relevant high-risk genes (28 samples for discovery phase, 87 and 408 samples for validation of non-synonymous variants and the recessive variant respectively). Cancer-free spouses of colorectal cancer patients served as controls (70 samples for the discovery phase, 284 and 293 samples for validation). They were recruited through the Swedish Colorectal Cancer Low-Risk Study. All 775 cases of breast cancer used for evaluating the recessive variant were collected from the Department of Clinical Genetics, Karolinska University Hospital. The 328 cases of sporadic breast cancer samples used in validation of non-synonymous variants were collected from a population-based cohort from Södersjukhuset, Stockholm. Genomic DNA was extracted from peripheral blood samples. Samples were genotyped using TaqMan SNP genotyping assay (Thermo Fisher Scientific, Waltham, Massachusetts.

Finnish validation cohorts. Rs16897117 was genotyped in two breast cancer cohorts from the Helsinki region, one including 1721 unselected cases and 755 additional familial cases ${ }^{18,31-33}$ and another consisting of 993 unselected cases $^{34}$, as well as in a cohort of 666 breast cancer patients from the Tampere region, described in detail previously ${ }^{31,33}$ (Table 1). CHEK2:c.1100delC genotype data were readily available from one of the Helsinki cohorts $^{35}$, the other two Finnish cohorts were genotyped for c.1100delC with a TaqMan assay.

BCAC data. The BCAC data used for final validation of the rs 16897117 was retrieved from the OncoArray project, described previously ${ }^{5}$. We included in the analysis the independent studies participating in the consortium, if there was sufficient data on reliably imputed c.1100delC available (at least 10 carrier cases and 10 healthy carrier controls per study). Only the study subjects with European ethnic background were included, and the Swedish and Finnish cohorts included in the discovery analyses were excluded. The selection yielded 13,767 breast cancer cases and 21,456 controls (Table 2).

Statistical analysis. Odds ratios, $95 \%$ confidence intervals and p-values were calculated to test the association with allele frequency using the DeFinetti programme provided as an online source ${ }^{36}$. The validation analyses were performed using R environment for statistical computing version 3.6.1 (R Core Team $(2019)^{37}$. For the case-only analysis of the Swedish and Finnish cohorts, a stratified Mantel-Haenszel odds ratio was estimated with R library epiDisplay ${ }^{38}$. The BCAC data analysis was performed with logistic regression. The interaction between c.1100delC and rs16897117 was assessed with likelihood-ratio test.

Ethics declaration. This study was approved by the Ethics Committee of Karolinska Institutet/Karolinska University Hospital. All individual studies, from which data was used, were approved by the appropriate medical ethical committees and/or institutional review boards. All methods were performed in accordance with the relevant guidelines and regulations. All study participants provided informed consent.

Received: 18 April 2021; Accepted: 28 June 2021

Published online: 20 July 2021

\section{References}

1. Antoniou, A. C. et al. Predicting the likelihood of carrying a BRCA1 or BRCA2 mutation: validation of BOADICEA, BRCAPRO, IBIS, Myriad and the Manchester scoring system using data from UK genetics clinics. J. Med. Genet. 45(7), 425-431 (2008).

2. Stratton, M. R. \& Rahman, N. The emerging landscape of breast cancer susceptibility. Nat. Genet. 40(1), 17-22 (2008).

3. Apostolou, P. \& Fostira, F. Hereditary breast cancer: the era of new susceptibility genes. Biomed. Res. Int. 2013, 747318 (2013). 
4. Mavaddat, N. et al. Polygenic risk scores for prediction of breast cancer and breast cancer subtypes. Am. J. Hum. Genet. 104(1), 21-34 (2019).

5. Michailidou, K. et al. Association analysis identifies 65 new breast cancer risk loci. Nature 551(7678), 92-94 (2017).

6. Fachal, L. et al. Fine-mapping of 150 breast cancer risk regions identifies 191 likely target genes. Nat. Genet. 52(1), 56-73 (2020).

7. Bartek, J., Falck, J. \& Lukas, J. CHK2 kinase: a busy messenger. Nat. Rev. Mol. Cell. Biol. 2(12), 877-886 (2001).

8. CHEK2 Breast Cancer Case-Control Consortium. CHEK2 ${ }^{*} 1100 \mathrm{delC}$ and susceptibility to breast cancer: a collaborative analysis involving 10,860 breast cancer cases and 9,065 controls from 10 studies. Am. J. Hum. Genet. 74(6), 1175-1182 (2004).

9. Weischer, M. et al. CHEK2* ${ }^{\star} 1100$ delC genotyping for clinical assessment of breast cancer risk: meta-analyses of 26,000 patient cases and 27,000 controls. J. Clin. Oncol. 26(4), 542-548 (2008).

10. Adank, M. A. et al. Excess breast cancer risk in first degree relatives of CHEK2 ${ }^{*} 1100$ delC positive familial breast cancer cases. Eur. J. Cancer 49(8), 1993-1999 (2013).

11. Weischer, M. et al. CHEK2*1100delC heterozygosity in women with breast cancer associated with early death, breast cancer-specific death, and increased risk of a second breast cancer. J. Clin. Oncol. 30(35), 4308-4316 (2012).

12. Fletcher, O. et al. Family history, genetic testing, and clinical risk prediction: pooled analysis of CHEK2* $1100 \mathrm{delC}$ in 1,828 bilateral breast cancers and 7,030 controls. Cancer Epidemiol. Biomark. Prevent. 18(1), 230-234 (2009).

13. Antoniou, A. C. \& Easton, D. F. Models of genetic susceptibility to breast cancer. Oncogene 25(43), 5898-5905 (2006).

14. Antoniou, A. C. et al. A comprehensive model for familial breast cancer incorporating BRCA1, BRCA2 and other genes. Br. J. Cancer 86(1), 76-83 (2002).

15. Muranen, T. A. et al. Genetic modifiers of CHEK2 ${ }^{\star} 1100 \mathrm{delC}$ associated breast cancer risk. Genet. Med. https://doi.org/10.1038/ gim.2016.147 (2016).

16. Kuchenbaecker, K. B. et al. Associations of common breast cancer susceptibility alleles with risk of breast cancer subtypes in BRCA1 and BRCA2 mutation carriers. Breast Cancer Res. 16(6), 3416 (2014).

17. Lee, A. et al. BOADICEA: a comprehensive breast cancer risk prediction model incorporating genetic and nongenetic risk factors. Genet. Med. 21(8), 1708-1718 (2019).

18. Vahteristo, P. et al. A CHEK2 genetic variant contributing to a substantial fraction of familial breast cancer. Am. J. Hum. Genet. 71(2), 432-438 (2002).

19. Zhang, H. et al. Genome-wide association study identifies 32 novel breast cancer susceptibility loci from overall and subtypespecific analyses. Nat. Genet. 52(6), 572-581 (2020).

20. Antoniou, A. C. et al. A locus on 19p13 modifies risk of breast cancer in BRCA1 mutation carriers and is associated with hormone receptor-negative breast cancer in the general population. Nat. Genet. 42(10), 885-892 (2010).

21. Couch, F. J. et al. Genome-wide association study in BRCA1 mutation carriers identifies novel loci associated with breast and ovarian cancer risk. PLoS Genet. 9(3), e1003212 (2013).

22. Antoniou, A. C. et al. Common alleles at $6 \mathrm{q} 25.1$ and $1 \mathrm{p} 11.2$ are associated with breast cancer risk for BRCA1 and BRCA2 mutation carriers. Hum. Mol. Genet. 20(16), 3304-3321 (2011).

23. Antoniou, A. C. et al. Common breast cancer-predisposition alleles are associated with breast cancer risk in BRCA1 and BRCA2 mutation carriers. Am. J. Hum. Genet. 82(4), 937-948 (2008).

24. Antoniou, A. C. et al. Common breast cancer susceptibility alleles and the risk of breast cancer for BRCA1 and BRCA2 mutation carriers: implications for risk prediction. Cancer Res. 70(23), 9742-9754 (2010).

25. Gaudet, M. M. et al. Identification of a BRCA2-specific modifier locus at $6 \mathrm{p} 24$ related to breast cancer risk. PLoS Genet. 9(3), e1003173 (2013).

26. Couch, F. J. et al. Identification of four novel susceptibility loci for oestrogen receptor negative breast cancer. Nat. Commun. 7 , $1-13(2016)$

27. Dunning, A. M. et al. Breast cancer risk variants at $6 \mathrm{q} 25$ display different phenotype associations and regulate ESR1, RMND1 and CCDC170. Nat. Genet. 48, 374-386 (2016).

28. Bojesen, S. E. et al. Multiple independent variants at the TERT locus are associated with telomere length and risks of breast and ovarian cancer. Nat. Genet. 45(4), 371-384 (2013).

29. Lawrenson, K. et al. Functional mechanisms underlying pleiotropic risk alleles at the $19 \mathrm{p} 13.1$ breast-ovarian cancer susceptibility locus. Nat. Commun. 7, 1-22 (2016).

30. Li, Y. et al. Resequencing of 200 human exomes identifies an excess of low-frequency non-synonymous coding variants. Nat. Genet. 42(11), 969-972 (2010).

31. Syrjäkoski, K. et al. Population-based study of BRCA1 and BRCA2 mutations in 1035 unselected Finnish breast cancer patients. J. Natl. Cancer Inst. 92(18), 1529-1531 (2000).

32. Kilpivaara, O. et al. Correlation of CHEK2 protein expression and c.1100delC mutation status with tumor characteristics among unselected breast cancer patients. Int. J. Cancer 113(4), 575-580 (2005).

33. Fagerholm, R. et al. $\mathrm{NAD}(\mathrm{P}) \mathrm{H}:$ quinone oxidoreductase $1 \mathrm{NQO1}{ }^{\star} 2$ genotype (P187S) is a strong prognostic and predictive factor in breast cancer. Nat. Genet. 40(7), 844-853 (2008).

34. Meretoja, T. J. et al. Pain at 12 months after surgery for breast cancer. JAMA 311(1), 90-92 (2014).

35. Muranen, T. A. et al. Genetic modifiers of CHEK2*1100delC-associated breast cancer risk. Genet. Med. 19(5), 599-603 (2017).

36. DeFinetti, programme https://ihg.gsf.de/cgi-bin/hw/hwal.pl.

37. R Core Team. R: a language and environment for statistical computing. R Foundation for Statistical Computing, Vienna, Austria. https://www.R-project.org/ (2019).

38. Virasakdi Chongsuvivatwong. epiDisplay: epidemiological data display package. R package version 3.5.0.1. https://CRAN.R-proje ct.org/package $=$ epiDisplay (2018).

\section{Acknowledgements}

We thank Anna Nurmi, MSc, for help with the genotyping and Research Nurse Outi Malkavaara with the patient data.

\section{Author contributions}

A.L. and C.W. conceived the study. X.J. and T.A.M. performed the data analyses and C.W, T.A.M, H.N. and A.L. the interpretation of the data. C.W., T.A.M., H.N. and A.L. drafted the manuscript. All authors contributed with data from their own studies, helped revise the manuscript, and approved the final version. All authors consented to this publication.

\section{Funding}

Financial support was provided through the regional agreement on medical training and clinical research (ALF) between the Stockholm County Council and Karolinska Institutet. The Helsinki study was supported by The Cancer Foundation Finland, The Sigrid Jusélius Foundation and The Helsinki University Hospital Research Fund. 


\section{Competing interests}

The authors declare no competing interests.

\section{Additional information}

Correspondence and requests for materials should be addressed to C.W.

Reprints and permissions information is available at www.nature.com/reprints.

Publisher's note Springer Nature remains neutral with regard to jurisdictional claims in published maps and institutional affiliations.

(c) (1) Open Access This article is licensed under a Creative Commons Attribution 4.0 International License, which permits use, sharing, adaptation, distribution and reproduction in any medium or format, as long as you give appropriate credit to the original author(s) and the source, provide a link to the Creative Commons licence, and indicate if changes were made. The images or other third party material in this article are included in the article's Creative Commons licence, unless indicated otherwise in a credit line to the material. If material is not included in the article's Creative Commons licence and your intended use is not permitted by statutory regulation or exceeds the permitted use, you will need to obtain permission directly from the copyright holder. To view a copy of this licence, visit http://creativecommons.org/licenses/by/4.0/.

(C) The Author(s) 2021 